Делитель потока зерна, теоретический анализ, каскадный делитель

Grain divider, theoretical analysis, cascade divider

\title{
ТЕОРЕТИЧЕСКОЕ ОБОСНОВАНИЕ КОНСТРУКТИВНЫХ ПАРАМЕТРОВ ДЕЛИТЕЛЯ С ГОРИЗОНТАЛЬНЫМ РАЗМЕЩЕНИЕМ ЩЕЛЕВЫХ ОТВЕРСТИЙ
}

К.Н. Тишанинов (фото)

к.т.Н., старший научный сотрудник лаборатории управления качеством технологических процессов в сельском хозяйстве А.В. Анашкин

к.Т.н., ведущий научный сотрудник лаборатории управления качеством технологических процессов в сельском хозяйстве ФГБНУ «Всероссийский научно-исследовательский институт использования техники и нефтепродуктов в сельском хозяйстве», г. Тамбов

Зерноочистительные агрегаты зачастую используют поточные технологические линии для послеуборочной очистки зерна, от качества работы которых зависит стоимость конечного продукта и рентабельность всего цикла производства зерна. Разделение потока зерна на две технологические линии происходит с использованием тройника зернопровода, что приводит к перегруженности одной линии и недогруженности другой. Зерноочистительные машины работают за рамками рекомендуемых нагрузок, что приводит к низкому качеству очистки и высокому уровню потерь зерна. Для качественной настройки зерноочистительных машин в составе технологии послеуборочной очистки зерна необходимы устройства для разделения потоков зерна. В ФГБНУ ВНИИТиН разработаны несколько классов авторегулируемых устройств (делителей): с грузовоспринимающей системой, с щелевыми отверстиями и т.д. В данной статье рассматривается одно из таких устройств - делитель потока зерна с горизонтальным размещением щелевых отверстий.

\section{Результаты исследований}

На предыдущих этапах исследований [1] нами была обоснована возможность создания авторегулируемого делителя потока зерна с горизонтальным размещением каскадных отверстий. Для обоснования конструктивных параметров делителя необходимо вычислить возможную погрешность деления при асимметричной подаче зерна в стабилизирующую ёмкость (рис. 1).

Как видно из рисунка 1, при стабилизации падающего потока зерна образуется насыпь, которую упрощённо можно представить в виде конуса. Расход зерна через отводы можно посчитать по следующей схеме (рис. 2). 


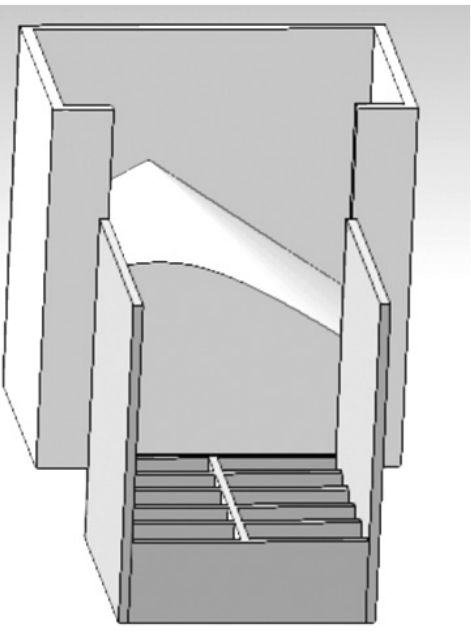

Рисунок 1 - Общий вид делителя при асимметричной подаче зерна

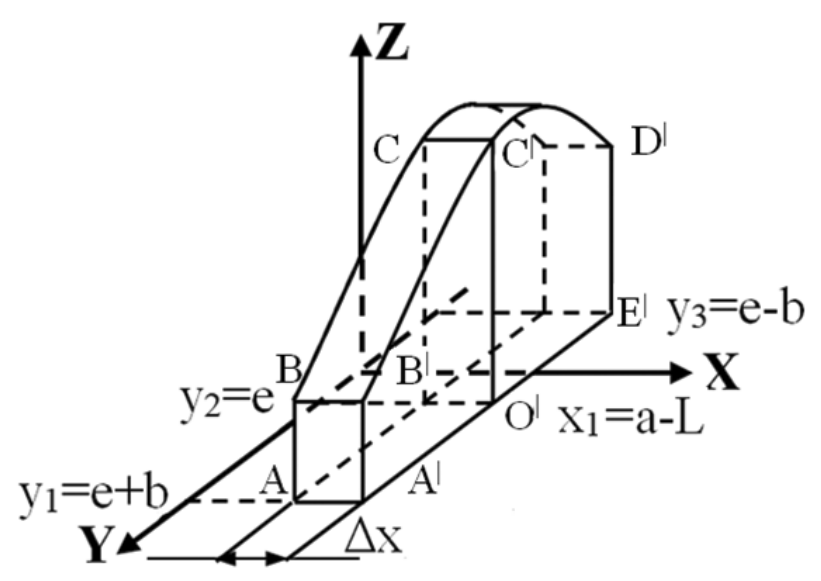

Рисунок 2 - Схема расчёта расходных характеристик отводов

Примем, что в первый отводящий канал (за элементарный промежуток времени $\Delta \mathrm{t}$ ) поступает объём зерна, равный площади фигуры OCDE $\left(\mathrm{S}_{1}\right)$, умноженный на элементарное расстояние отводящего канала $\Delta \mathrm{x}$. Тогда расход зерна первого отводящего канала $\left(\mathrm{V}_{1}\right)$ вычисляется по формуле:

$$
V_{1}=\frac{s_{1} \cdot \Delta x}{\Delta t} .
$$

Аналогично найдём расход зерна через второй отводящий канал $\left(\mathrm{V}_{2}\right)$ :

$$
V_{2}=\frac{s_{2} \Delta x}{\Delta t}
$$

где $\mathrm{S}_{2}$ - площадь фигуры $\mathrm{ABCO}$, мм² $^{2}$.

Тогда погрешность деления $(\Delta)$ можно вычислить как:

$$
\Delta=\frac{V_{1}-V_{2}}{V_{1}}=\frac{\frac{S_{1} \cdot \Delta x}{\Delta t}-\frac{S_{2} \cdot \Delta x}{\Delta t}}{\frac{S_{1} \cdot \Delta x}{\Delta t}}=\frac{S_{1}-S_{2}}{S_{1}},
$$

где $\mathrm{S}_{1}$ - площадь фигуры, ограниченная:

кривой $\mathrm{z}(\mathrm{y})=\mathrm{H}-\operatorname{tg}(\alpha) \cdot \sqrt{(L-a)^{2}+y^{2}}$, (3)

прямой $\mathrm{z}(\mathrm{y})=0$,

прямой $\mathrm{z}(\mathrm{y})=\mathrm{e}-\mathrm{b}$,

прямой $\mathrm{z}(\mathrm{y})=\mathrm{e}$,

где Н - высота насыпи, м;

$\alpha$ - угол естественного откоса, град;

$2 \mathrm{a}$ - длина стабилизирующей ёмкости, м;

$\mathrm{L}$ - величина горизонтального (по длине ёмкости) отклонения от симметричной подачи зерна, м;

$2 \mathrm{~b}$ - ширина стабилизирующей ёмкости, м;

е - величина горизонтального (по ширине ёмкости) отклонения от симметричной подачи зерна, м.

Следовательно, площадь фигуры $\mathrm{S}_{1}$ можно вычислить как:

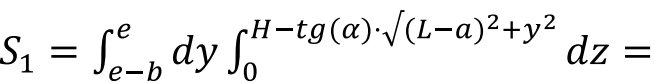

$=\int_{e-b}^{e}\left(H-\operatorname{tg}(\alpha) \cdot \sqrt{(L-a)^{2}+y^{2}}-0\right) d y=$

$=H(e-e+b)-\operatorname{tg}(\alpha) \cdot\left(e \cdot \sqrt{(L-a)^{2}+e^{2}}-\right.$

$\left.-(e-b) \cdot \sqrt{(L-a)^{2}+(e-b)^{2}}\right) / 2-\operatorname{tg}(\alpha) \cdot$

$(L-a)^{2} \cdot\left(\ln \left(|e|+\sqrt{\left.(L-a)^{2}+e^{2}\right)-}\right.\right.$

$\left.-\ln \left(|e-b|+\sqrt{(L-a)^{2}}+(e-b)^{2}\right)\right) / 2$.

Аналогичным образом найдём площадь фигуры $\mathrm{S}_{2}$ :

$S_{2}=\int_{e}^{e+b} d y \int_{0}^{H-\operatorname{tg}(\alpha) \cdot \sqrt{(L-a)^{2}+y^{2}}} d z=$

$=\int_{e}^{e+b}\left(H-\operatorname{tg}(\alpha) \cdot \sqrt{(L-a)^{2}+y^{2}}-0\right) d y=$

$=H(e-e+b)-\operatorname{tg}(\alpha) \cdot((e+b) \cdot$

$\cdot \sqrt{(L-a)^{2}+(e+b)^{2}}$

$\left.-e \cdot \sqrt{(L-a)^{2}+e^{2}}\right) / 2-\operatorname{tg}(\alpha) \cdot$

$\cdot(L-a)^{2} \cdot\left(\ln \left(|e+b|+\sqrt{(L-a)^{2}+(e+b)^{2}}\right)-\right.$

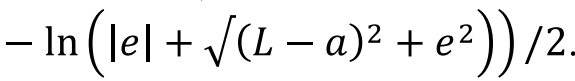

Теоретический анализ возможной погрешности разделения потока зерна $(\Delta)$ проводился при следующих варьируемых параметрах (табл. 1):

Результаты теоретического анализа показали, что величина (b) слабо влияет на максимальную погрешность разделения. С уменьшением высоты насыпи (Н) с 60 см до 20 см погрешность разделения возрастает (рис. 3). 
Таблица 1 - Факторы варьирования

\begin{tabular}{|c|c|c|c|c|}
\hline $\begin{array}{c}\text { Наименование фактора } \\
\text { варьирования }\end{array}$ & $\begin{array}{c}\text { Отклонение } \\
\text { потока по длине } \\
\text { ёмкости (L) }\end{array}$ & $\begin{array}{c}\text { Отклонение потока по } \\
\text { ширине ёмкости (e) }\end{array}$ & Ширина канала (2b) & Высота насыпи (H) \\
\hline Интервал варьирования & $0 . .25 \%(\mathrm{a})$ & $0 . .15 \mathrm{~cm}$ & $10,20,30 \mathrm{~cm}$ & $30,45,60 \mathrm{~cm}$ \\
\hline
\end{tabular}

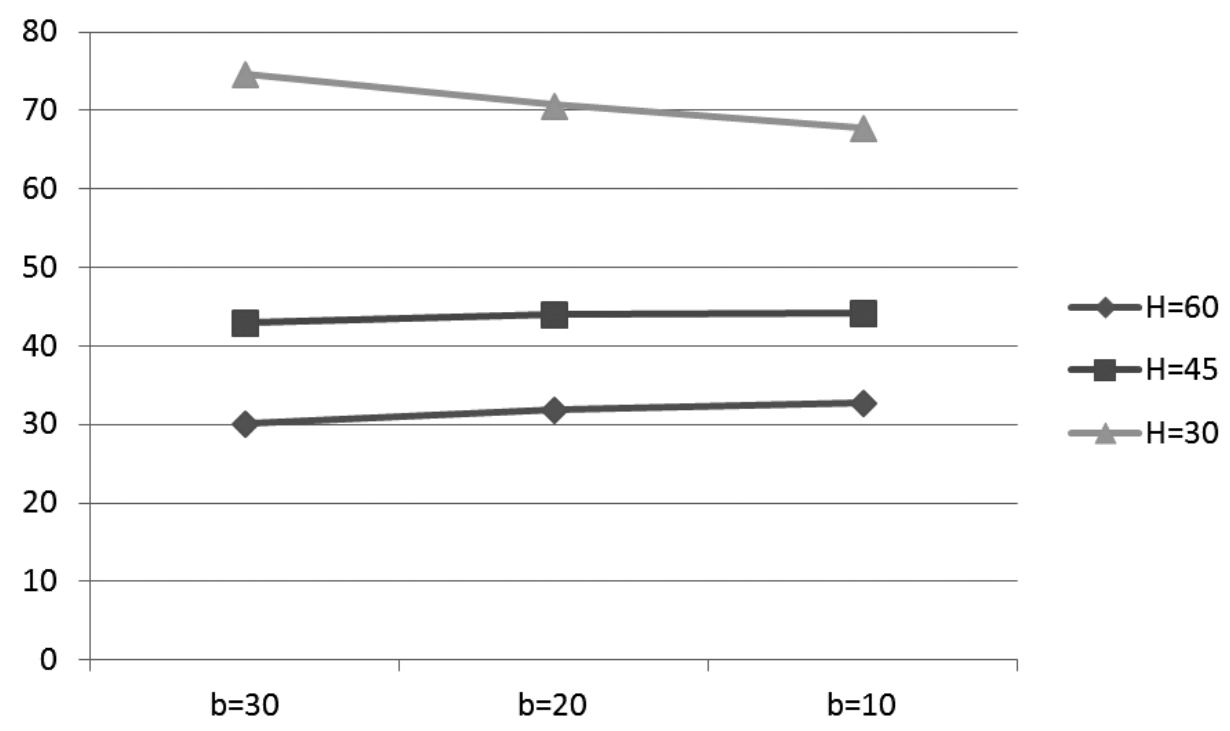

Рисунок 3 - Зависимость максимальной погрешности деления $\left(\Delta_{\max }\right)$ от ширины канала (2b) и высоты насыпи $(\mathrm{H})$

При малых значениях отклонения потока по ширине ёмкости (величина е $=1,5 \mathrm{~cm}$ ) и малых значения (L) с уменьшением ширины отводящих каналов с 60 см до 20 см погрешность разделения увеличивается с 3,9 до 6,4\% (в 1,64 раза) (рис. 4).
Как видно из рисунка 4, возрастание погрешности деления происходит только при малых значениях (L). При больших значениях (L) погрешность разделения намного меньше и уменьшается вместе с уменьшением ширины

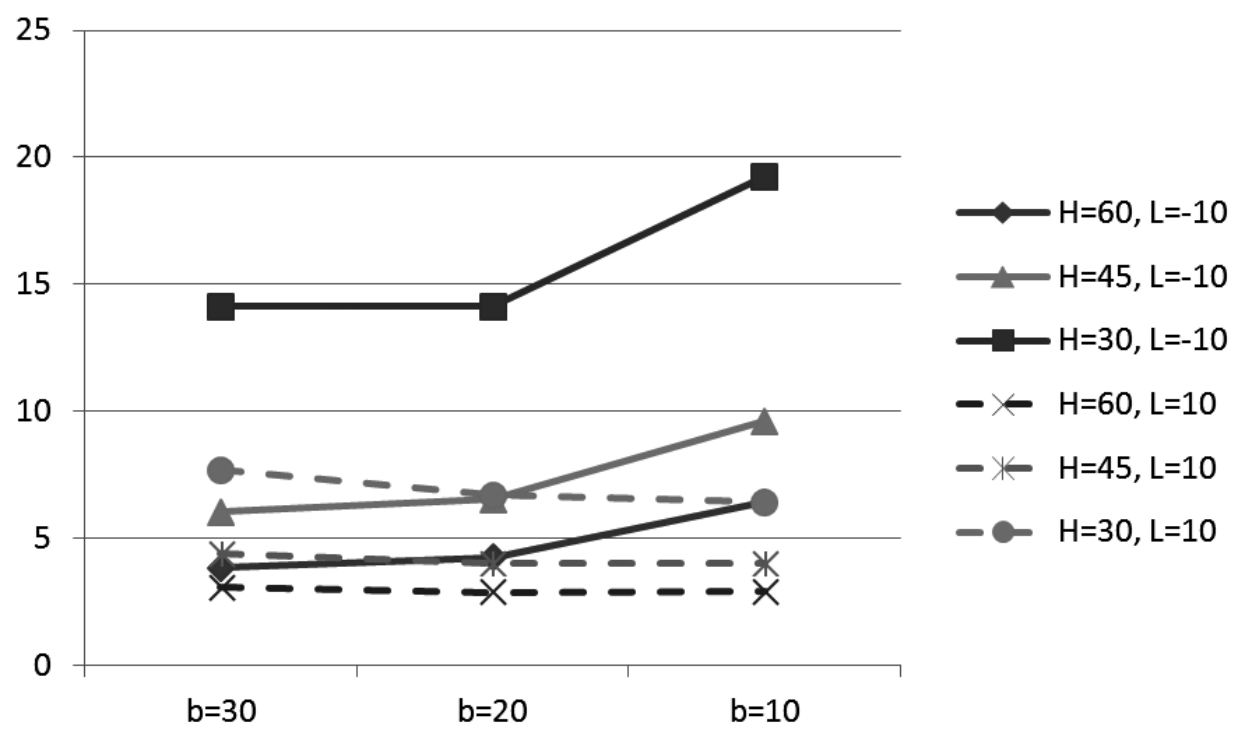

Рисунок 4 - Зависимость погрешности деления $(\Delta)$ при малых значениях отклонения подачи по ширине ёмкости $(e=1,5)$ от ширины канала $(2 b)$ при переменных значениях высоты насыпи $(\mathrm{H})$ и отклонений подачи по длине ёмкости (L) 
канала (2b). Учитывая, что высота насыпи - величина переменная, и она зависит от подачи зерна в стабилизирующую ёмкость, то снизить общую погрешность можно, приняв минимальные (в выбранном диапазоне варьирования) значения ширины канала и максимальные значения отклонения подачи по длине ёмкости ( $\mathrm{L}=10,2 \mathrm{~b}=20)$.

С увеличением отклонения потока зерна по ширине стабилизирующей ёмкости погрешность разделения резко возрастает (рис. 5).

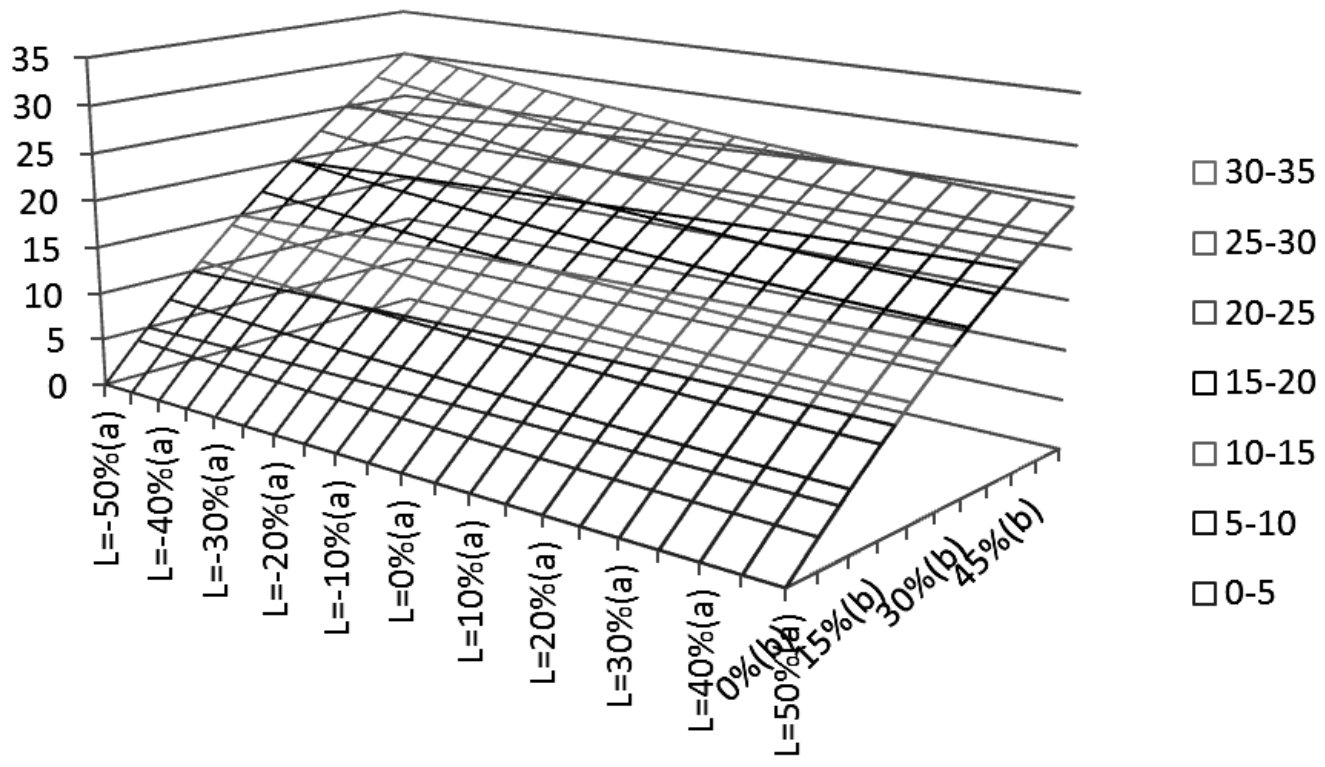

Рисунок 5 - Зависимость погрешности деления $(\Delta)$ от значений отклонения подачи по ширине ёмкости (е) и длине ёмкости (L) при фиксированных значениях высоты насыпи (H=60 cм) и ширины канала $(2 \mathrm{~b}=60)$

\section{Выводы}

При фиксированных значениях отклонения потока по ширине ёмкости (е) наименьшие значения погрешности деления потока $(\Delta)$ наблюдаются при минимальной ширине отводящего канала $(2 \mathrm{~b}=20 \mathrm{~cm})$, максимальном значении отклонения потока по длине ёмкости ( $\mathrm{L}=10$ см) и максимальной высоте насыпи (H = 60 см). Учитывая, что высота насыпи (Н) - величина переменная, зависящая от подачи зерна в стабилизирующую ёмкость, то основной рекомендацией при проектировании делителя с горизонтальным расположением каскадных отверстий будет центральная подача зерна по ширине ёмкости, в сторону к передней стенке, и минимально допустимое значение ширины отводящих каналов (10..20 см).

\section{Лumepamypa}

1. Анашкин, А.В. Морфологический анализ устройств для управления массовыми потоками зерна [Текст] / А.В. Анашкин, К.Н. Тишанинов // Наука в центральной России. - 2019. - № 1 (37). - С. 11-19.

\section{References}

1. Anashkin, A.V. Morfologicheskij analiz ustrojstv dlja upravlenija massovymi potokami zerna [Tekst] / A.V. Anashkin, K.N. Tishaninov // Nauka v central'noj Rossii. - 2019. - № 1 (37). - S. 11-19. 